\title{
Fabrication of Fe-based ribbon with controlled permeability by Joule heating under tensile stress
}

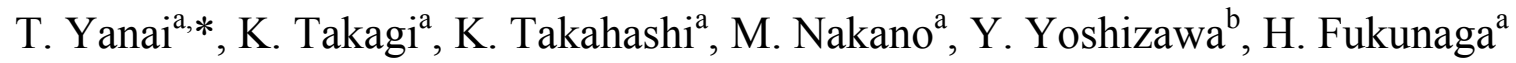 \\ ${ }^{a}$ Department of Electrical and Electronic Engineering, Faculty of Engineering of the University of Nagasaki, Nagasaki, 852-8521, Japan \\ ${ }^{b}$ Advanced Electronics Research Laboratory, Hitachi Metals Ltd., Kumagaya, 360-0843, Japan
}

Elsevier use only: Received 30 Augasut 2007; revised 20 Junuary 2008; accepted date here

\begin{abstract}
We prepared an Fe-based ribbon with controlled permeability and low magnetic loss by Continuous Stress-Annealing by Joule Heating (CSA-JH) method. The shortest annealing time necessary for the obtaining of the ribbons with completely developed anisotropy was 1 sec, but in order to obtain the sufficiently crystallized ribbons with small saturation magnetostriction it is required to perform annealing longer than 2 sec. A toroidal core was prepared from a long ribbon with controlled permeability and small saturation magnetostriction, and the magnetic loss and relative permeability of the core were evaluated in the frequency range of $0.1-3 \mathrm{MHz}$. The permeability was kept constant up to $2 \mathrm{MHz}$, and the magnetic loss is lower than that for other types of core with a similar permeability value. These results suggest that the CSA-JH method is a promising method for realizing an Fe-based core with excellent magnetic properties.
\end{abstract}

(C) 2008 Elsevier B.V. All rights reserved

PACS: $51.60 .+\mathrm{a} ; 75.30 . \mathrm{Gw} ; 85.70 .-\mathrm{w}$

Keywords: Continuous stress-annealing; Stress-induced anisotropy; Joule heating; Controlled permeability; Nanostructure material

\section{Introduction}

In order to advance high-density-packaging and energy saving of electronic devices, size reduction and improvement in efficiency of magnetic cores are strongly required. Although an Fe-based core prepared from a long ribbon with uniaxial anisotropy developed by stress-annealing [1-4] shows excellent magnetic properties compared with conventional cores with a similar permeability [5-8], the simplification of the annealing process such as reduction in annealing time is required for mass production.

For this purpose, we have proposed the Continuous StressAnnealing by Joule-Heating method $[9,10]$. In this study, the ribbons were prepared by this method focusing on reduction in annealing time and application to toroidal core.

\section{Experimental procedure}

\subsection{Annealing for development of anisotropy}

Nanocrystalline Fe-based ribbons with a uniaxial anisotropy perpendicularly to the ribbon-axis were fabricated by Jouleheating amorphous $\mathrm{Fe}_{73.5} \mathrm{Cu}_{1} \mathrm{Nb}_{3} \mathrm{Si}_{15.5} \mathrm{~B}_{7}$ ones (Hitachi Metals Ltd., 120 or $500 \mathrm{~mm}$ in length, $2 \mathrm{~mm}$ in width and $20 \mu \mathrm{m}$ in thickness) in air under the tensile stress of $100 \mathrm{MPa}$, referred as the CSA-JH (Continuous Stress-Annealing by Joule Heating) method. The apparatus for the CSA-JH method was described elsewhere [9]. The supplied current density $j$ to the amorphous ribbon was varied from 32.5 to $42.5 \mathrm{~A} / \mathrm{mm}^{2}$. The annealing time $t_{a}$ was calculated from the distance between electrodes $l_{e}(=35 \mathrm{~mm})$ and the moving velocity of the ribbon $v_{m}$ by eq(1).

$$
t_{a}=l_{e} / v_{m}
$$

\subsection{Measurements}

\subsubsection{Hysteresis loop}

The dc-hysteresis loop of the annealed ribbon was traced

* Corresponding author. Tel.: +81-95-819-2554; fax: +81-95-819-2554.

E-mail address: t-yanai@nagasaki-u.ac.jp. 
with a computer-aided B-H loop tracer (Riken BHS-40, $H_{m}=$ $4000 \mathrm{~A} / \mathrm{m}, f=50 \mathrm{mHz}$ ). The uniaxial anisotropy energy constant $K_{u}$ was obtained by numerical integration of $H \bullet \Delta I$ in the first quadrant of the loop.

\subsubsection{Estimation of degree of crystallization}

For evaluation of the crystallization state in annealed ribbons, thermal analysis was carried out with a differential scanning calorimeter (Seiko Instruments DSC-6200) in $\mathrm{Ar}$ flow. The heating rate was fixed at $10^{\circ} \mathrm{C} / \mathrm{min}$ during an analysis. Figure 1 shows exothermal curves of an amorphous and an annealed ribbon. The exothermic heat peak around 540 ${ }^{\circ} \mathrm{C}$ indicates development of crystallization. The degree of crystallization $p$ was estimated by

$$
p=\left(S_{\text {Amor }}-S_{\text {Anneal }}\right) / S_{\text {Amor }} \times 100,
$$

where $S_{\text {Amor }}$ and $S_{\text {Anneal }}$ are the area of the heat peaks of the amorphous and the annealed ribbon, respectively. $S_{\text {Amor }}$ and $S_{\text {Anneal }}$ were obtained by numerical integration of the exothermal curve between 500 and $580{ }^{\circ} \mathrm{C}$.

\subsubsection{Elongation}

The elongation of the annealed ribbon, $\Delta L / L$, was calculated from

$$
\Delta L / L=\left(L^{\prime}-L\right) / L \times 100
$$

where $L$ and $L$, are the length of the ribbon before and after stress-annealing, respectively.

\subsubsection{Ac-magnetic properties of toroidal core}

The annealed $500 \mathrm{~mm}$-long ribbon was formed into a toroidal core with a ceramic bobbin. The relative permeability $\mu_{r}$ and the magnetic loss per cycle $P_{c v} / f$ of the prepared core at $B_{m}=0.1 \mathrm{~T}$ were evaluated with a B-H analyzer (Iwatsu SY8232 ) in the frequency $f$ range from 0.1 to $3 \mathrm{MHz}$.

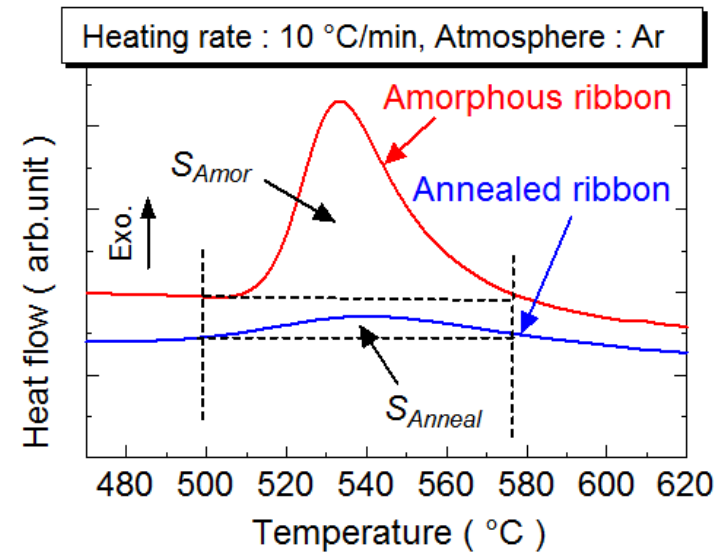

Fig.1 Exothermal curves of amorphous and annealed ribbons. The exothermic heat flow around $540{ }^{\circ} \mathrm{C}$ indicates development of crystallization.

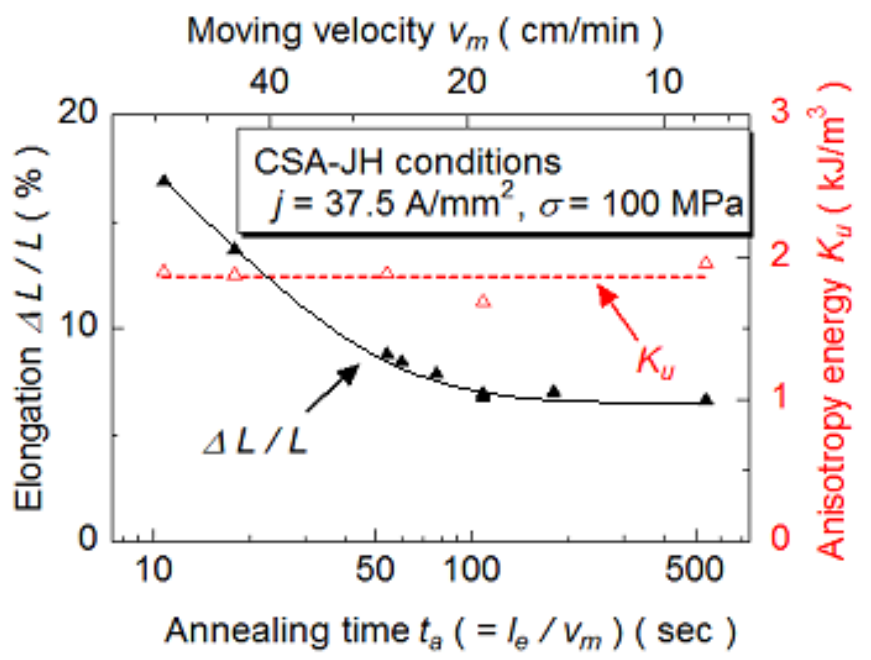

Fig.2 Elongation $\Delta L / L$ and anisotropy energy $K_{u}$ of annealed ribbons as a function of annealing time $t_{a}$.

\section{Result and discussion}

\subsection{Dependence of elongation and of anisotropy energy on annealing time}

Figure 2 shows the dependence of elongation $\Delta L / L$ and of anisotropy energy $K_{u}$ on annealing time $t_{a}$. As seen in the figure, $K_{u}$ did not depend on $t_{a}$ in the wide range of $t_{a}$ from 10 to $500 \mathrm{sec}$. On the other hand, $\Delta L / L$ is large at $t_{a} \leq 50 \mathrm{sec}$. This phenomenon suggests that the large elongation, which is observed at $t_{a} \leq 50 \mathrm{sec}$, did not affect a magnitude of $K_{u}$. As a wasteful elongation has a tendency of mechanical break of the ribbon during annealing, we need to pay attention to restrain the wasteful elongation.

\subsection{Reduction in annealing time}

Figure 3 shows a relationship among the development states of anisotropy, current density $j$ and annealing time $t_{a}$, together with the result for $\mathrm{JH}$ method [11]. The $\mathrm{JH}$ method corresponds $v_{m}=0 \mathrm{~cm} / \mathrm{min}$ for the CSA-JH method. The development states of anisotropy were categorized into 5 ones from hysteresis loops of annealed ribbons. Symbols, " $\circ$ ", " $\Delta$ ", "/", "+", and "x", indicate "completely developed", "under development", "not developed", "magnetically-deteriorated", and "mechanically broken during annealing", respectively. The completely developed anisotropy could be obtained stably in the range of $t_{a}=1-200 \mathrm{sec}$ at $j=37.5 \mathrm{~A} / \mathrm{mm}^{2}$. In our equipment, the shortest annealing time $t_{a m i n}$ which enabled the fabrication of a ribbon with completely developed anisotropy, was approximately $1 \mathrm{sec}$. This value of $t_{\text {amin }}$ was almost the same as that for the $\mathrm{JH}$ method one $(0.5 \mathrm{sec})$.

When an annealed ribbon is formed into a toroidal core, mechanical stress induces a magnetic anisotropy through its magnetostriction. As this anisotropy may cause deterioration 


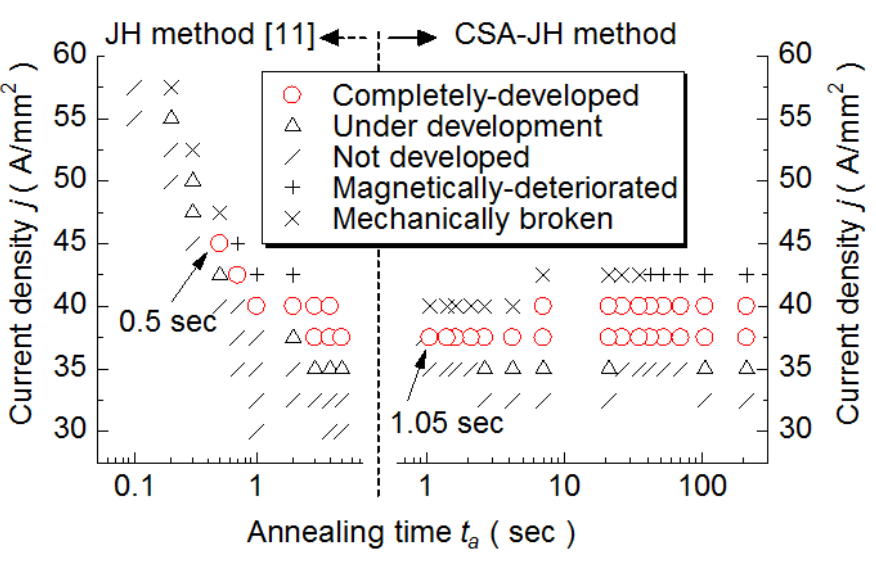

Fig.3 Relationship among development state of anisotropy, current density $j$ and annealing time $t_{a}$. "०", " $\Delta$ ", “", “+", and " $\times "$ indicate "completely developed", "under development", "not developed", "Magneticallydeteriorated", and "mechanically-broken during annealing", respectively.

of magnetic properties, the annealed ribbon is desired to have a small saturation magnetostriction value. As a magnetostriction in the sufficiently crystallized $\mathrm{Fe}_{73.5} \mathrm{Cu}_{1} \mathrm{Nb}_{3} \mathrm{Si}_{15.5} \mathrm{~B}_{7}$ ribbon is nearly zero [12-14], sufficiently crystallization is important for size reduction of the cores with excellent magnetic properties. Thus, we investigated the crystallization state for the annealed ribbons.

Figure 4 shows exothermal curves of ribbons prepared at $t_{a}$ $=1.05,1.4$ and $2.1 \mathrm{sec}$. Although the exothermic heat flow was observed in the ribbons with $t_{a}=1.4 \mathrm{sec}$ and $1.05 \mathrm{sec}$, the flow did not happen in the annealed one with $t_{a}=2.1 \mathrm{sec}$. This result suggests that the ribbon prepared at $t_{a} \leq 1.4 \mathrm{sec}$ deteriorates their magnetic properties during formation of a toroidal core.

The shortest annealing time which enables us to obtain a ribbon crystallized sufficiently was $2.1 \mathrm{sec}$ in our equipment. This value is $25 \%$ as short as that for Continuous StressAnnealing with a Furnace (CSA-F) method [15].

\subsection{Magnetic properties of toroidal core}

As we confirmed that the ribbon prepared under a short annealing time of $2.1 \mathrm{sec}$ was crystallized sufficiently and has anisotropy developed completely, a toroidal core with the inner diameter $D_{\text {in }}$ of $15 \mathrm{~mm}$ was prepared and its ac magnetic properties were evaluated.

Figure 5 shows the frequency dependencies of relative permeability $\mu_{r}$ (a) and magnetic loss per cycle $P_{c v} f(b)$ of the prepared core, respectively. Magnetic losses at $f=0.1 \mathrm{MHz}$ for conventional cores with a similar permeability reported by Endo et al. [16] are also shown in the figure 5 (b). The prepared core had kept the permeability constant up to $2 \mathrm{MHz}$ and the low value of magnetic loss compared with those for the conventional ones. These properties were almost the same as those for the previously reported ones [5].

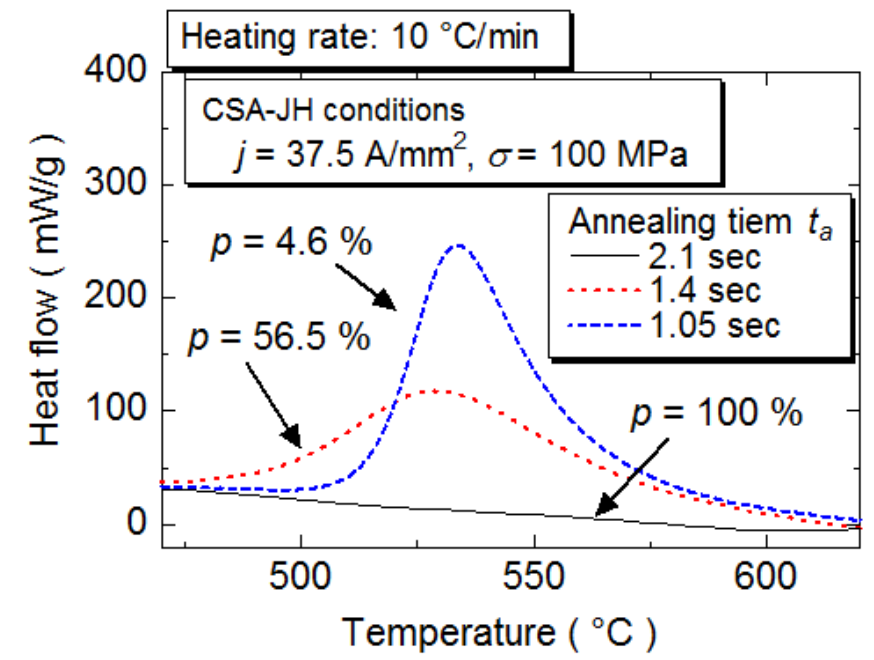

Fig.4 Exothermal curves of ribbons prepared at $t_{a}=1.05,1.4$ and $2.1 \mathrm{sec}$. The measurement was carried out in a temperature range from 470 to $620^{\circ} \mathrm{C}$.
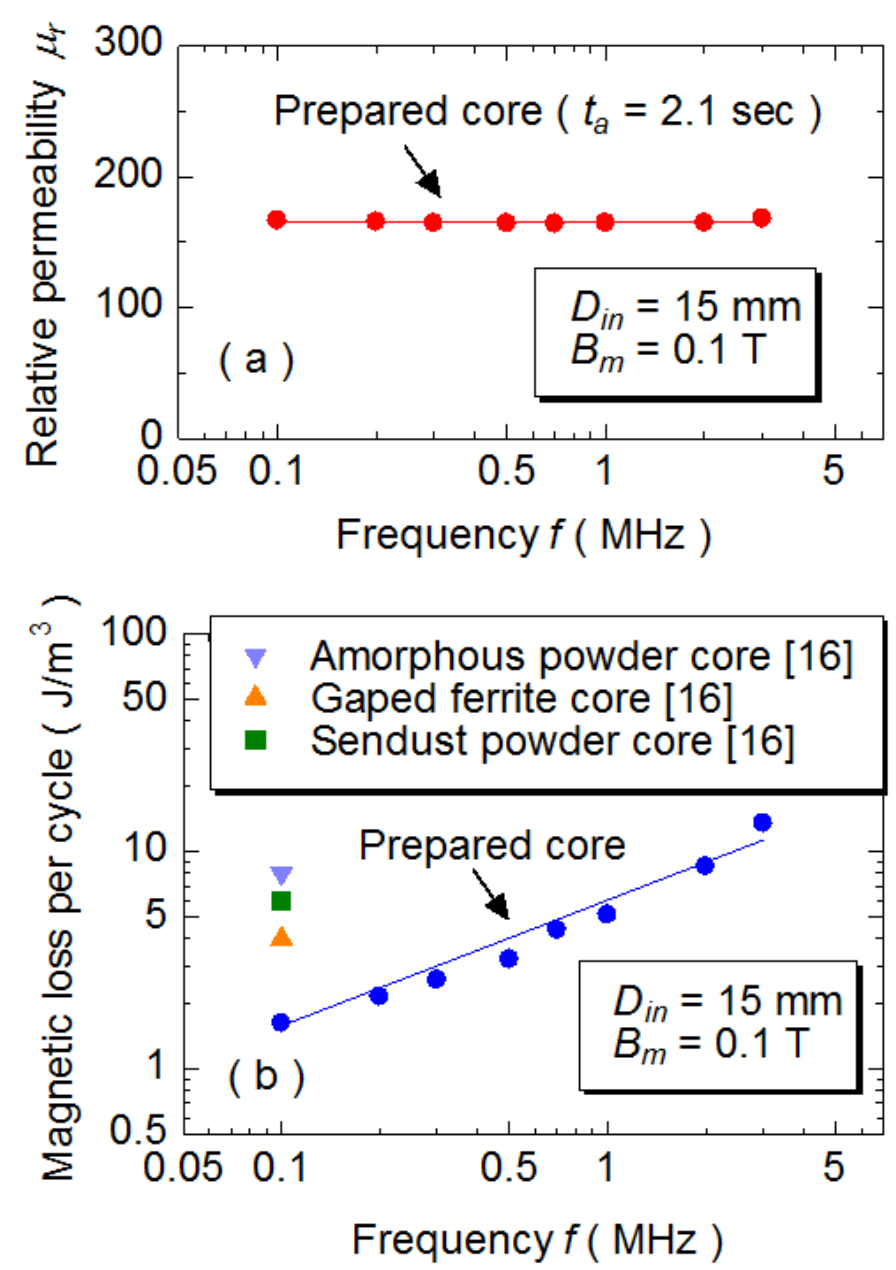

Fig.5 Relative permeability $\mu_{r}$ (a) and magnetic loss per cycle $P_{c v} / f$ (b) of a developed core as a function of frequency. Magnetic losses at $f=0.1 \mathrm{MHz}$ for different types of cores with a similar permeability were shown in the Fig. 5 (b) $[16]$. 


\section{Conclusion}

We prepared the Fe-based ribbons sufficiently nanocrystallized with controlled permeability focusing on reduction in annealing time and the application to a toroidal core by the CSA-JH method. The obtained results are summarized as follows;

(1) We achieved a reduction in an annealing time compared with that of the CSA-F method. Resultantly, we can obtain an Fe-based ribbon with completely developed anisotropy and small saturation magnetostriction in a short time of $2.1 \mathrm{sec}$.

(2) A toroidal core prepared from the long ribbon with small magnetostriction value had constant permeability up to 2 $\mathrm{MHz}$ and showed lower magnetic loss than those for different types of cores with a similar permeability.

\section{References}

[1] L. Kraus, K. Závěta, O. Heczko, P. Duhaj, G. Valsák and J. Schneider, J. Magn. Magn. Mater., vol. 112, pp. 275-277, 1992.

[2] N. Murillo, J. González, J. M. Blanco and Vázquez, J. Appl. Phys., vol. 74, pp. 3323-3327, 1993.
[3] G. Herzer, IEEE Trans. Magn., vol. 30, pp. 4800-4802, 1994.

[4] B. Hofmann and H. Kronmüller, Nanostructure Mater., vol. 6, pp. 961964, 1995.

[5] H. Fukunaga, N. Furukawa, H. Tanaka, and M. Nakano, J. Appl. Phys., vol. 87, pp. 7103-7105, 2000.

[6] H. Fukunaga, H. Tanaka, T. Yanai, M. Nakano, K. Takahashi, Y. Yoshizawa, K. Isiyama, and K. Arai, J. Magn. Magn. Mater., vol. 242245, pp. 279-281, 2002.

[7] H. Fukunaga, T. Yanai, H. Tanaka, M. Nakano, K. Takahashi, Y. Yoshizawa, K. Ishiyama and K. I. Arai, IEEE Trans. Magn., vol. 38, pp. 3138-3140, 2002.

[8] T. Yanai, M. Yamasaki K. Takahashi, M. Nakano Y. Yoshizawa and H. Fukunaga, IEEE Trans. Magn., vol. 40, pp. 2721-2723, 2004.

[9] T. Yanai, A. Shimada, K. Takahashi, M. Nakano, Y. Yoshizawa and H. Fukunaga, IEEE Trans. Magn., vol. 42, pp.2781-2783, 2006.

[10] T. Yanai,A. Shimada, K. Takagi, K. Takahashi, M. Nakano, and H. Fukunaga, Y. Yoshizawa, J. Appl. Phys., vol. 101, 09N110, 2007.

[11] T. Yanai, M. Yamasaki, K. Takahashi, M. Nakano, H. Fukunaga and Y. Yoshizawa, Conf. Proc. of SMM 16, vol. 2, pp. 737-741, 2004.

[12] K. Twarowski, M. Kuźmiński, A. Ślawska-Waniewska, H.K. Lachowicz and G. Herzer, J. Magn. Magn. Mater., vol. 140-144, pp. 449-450, 1995.

[13] K. Twarowski, M. Kuźmiński, A. Ślawska-Waniewska, H.K. Lachowicz and G. Herzer, J. Magn. Magn. Mater., vol. 150, pp. 85-92, 1995.

[14] L. Kraus, V. Hašlar, O. Heczko and K. Závěta, J. Magn. Magn. Mater., vol. 157-158, pp. 151-152, 1996.

[15] T. Yanai, T. Ohya, K. Takahashi, M. Nakano and H. Fukunaga, J. Magn. Magn. Mater., vol. 290-291, pp. 1502-1505, 2005.

[16] I. Endo, H. Tatsumi, I. Otsuka, H. Yamamoto, A. Shintani, H. Koshimoto, M. Yagi and K. Murata, IEEE Trans. Magn., vol. 36, pp. 3421-3423, 2000. 